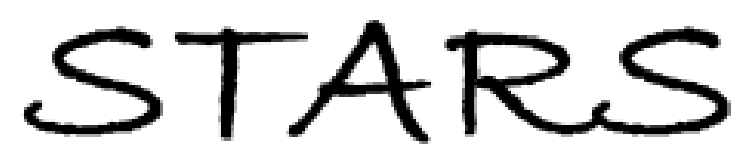

University of Central Florida

STARS

2016

\title{
Transitions in Polyamorous Identity and Intercultural Communication: An Application of Identity Management Theory
}

\author{
Allison Table \\ University of Central Florida
}

Part of the Interpersonal and Small Group Communication Commons

Find similar works at: https://stars.library.ucf.edu/etd

University of Central Florida Libraries http://library.ucf.edu

This Masters Thesis (Open Access) is brought to you for free and open access by STARS. It has been accepted for inclusion in Electronic Theses and Dissertations, 2004-2019 by an authorized administrator of STARS. For more information, please contact STARS@ucf.edu.

\section{STARS Citation}

Table, Allison, "Transitions in Polyamorous Identity and Intercultural Communication: An Application of Identity Management Theory" (2016). Electronic Theses and Dissertations, 2004-2019. 4883.

https://stars.library.ucf.edu/etd/4883

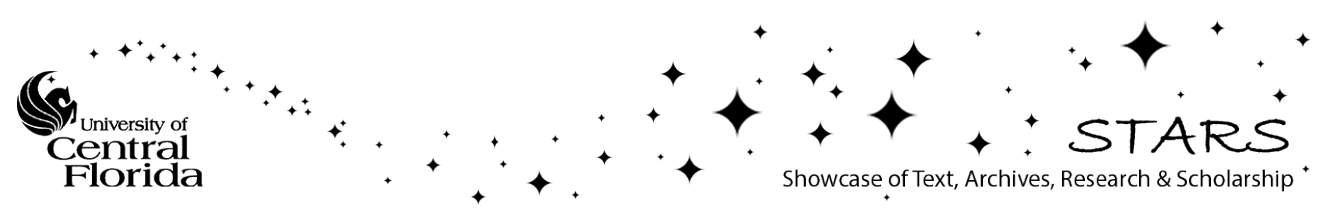




\section{TRANSITIONS IN POLYAMOROUS IDENTITY AND INTERCULTURAL COMMUNICATION: AN APPLICATION OF IDENTITY MANAGAMENT THEORY}

ALLISON TABLE

B.S. Florida Gulf Coast University, 2012

A thesis submitted in partial fulfillment of the requirements for the degree of Master of Arts in the Nicholson School of Communication in the College of Sciences at the University of Central Florida Orlando, Florida 
(C) 2016 Allison Table 


\begin{abstract}
This thesis examines the intercultural communicative strategies and non-monogamous identity formation of polyamorous individuals. The purpose of this study is to investigate the identity management tactics that polyamorous individuals utilize to navigate a society in which monogamy is the norm. Interactions of polyamorous individuals within a monoganormative society are treated as a type of intercultural communication due to the contrasting cultural identities and communication rules. E-interviews with 38 polyamorous individuals provided basis for phone and Skype interviews. The Retrospective Interview Technique (Huston, Surra, Fitzgerald, \& Cate, 1981) required interviewees to recall development of their polyamorous identity and interactions with monogamous others from the past to present day. Twenty-two interviews produced the phases of identity management, including Trial and Error, Enmeshment (Mixing Up), and Renegotiation. Analyzing interviewees' communication also produced the themes of managing stigma and impressions of the relationship identity. Implications of the study include a richer understanding of polyamorous identity management, polyamory as a relationship orientation, and a new perspective in applying identity management theory.
\end{abstract}




\section{ACKNOWLEDGMENTS}

Many thanks first to my family and friends who have been by my side every step of the way. First, my parents Susan and Patrick, you have been encouraging and proud beacons of inspiration - always accepting, always patient, and forever supportive of my creative and academic endeavors. To my partner McCarty, for your love, humor, and support as I work my way through this process. I love you, and thank you. And to my friends in the program (The Cohort), my dance troupe, and all over the country who have continually cheered me on and made the tough times bearable and the great times into joyful memories - I don't think I would have made it through grad school and the thesis without you. Talking out my lofty ideas with you all and turning them from fever dreams to reality has never made me feel more nurtured as a scholar, performer, researcher, and human.

To my thesis chair Harry Weger, for your support and guidance. Even though we're so different in our approaches, you mentored me with countless opportunities to grow. I feel very fortunate to have had you as my coach. Also, to committee members Sally Hastings and Jennifer Sandoval for providing feedback and believing in my abilities every step of the way. I cannot thank you enough for your time, investment in me, words of encouragement, and real talk during the thesis process and in my two years of grad school. I believe I am a stronger scholar and a stronger person for knowing you.

Thank you to all of the interviewees who participated in this study. I appreciate you so much for enthusiastically sharing insight, intimate feelings, and life experiences regarding your identity and relationships. Your voices made this project possible. 


\section{TABLE OF CONTENTS}

CHAPTER 1: INTRODUCTION ........................................................................... 1

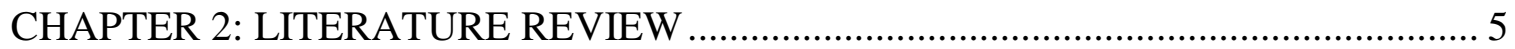

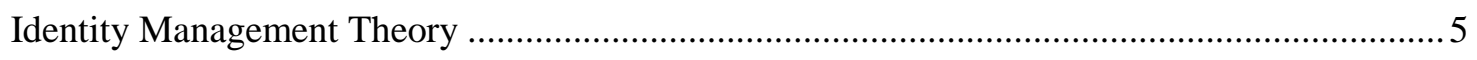

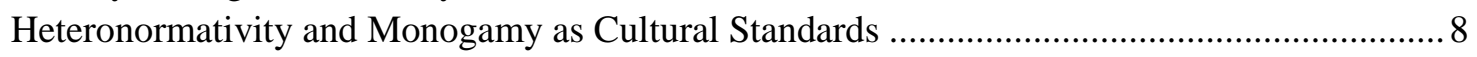

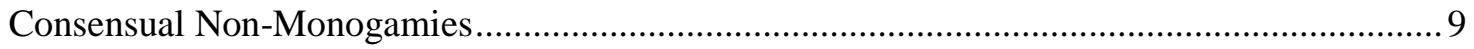

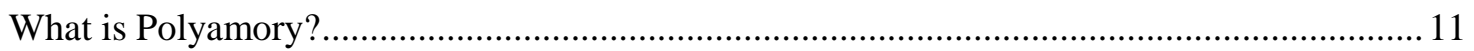

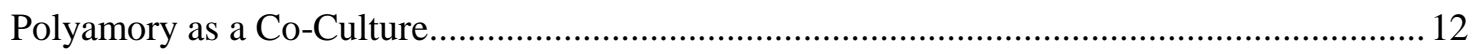

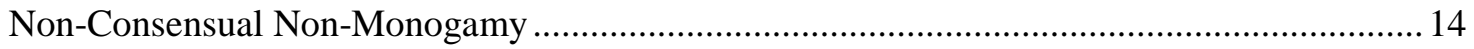

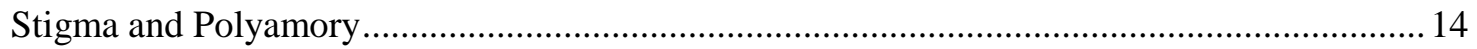

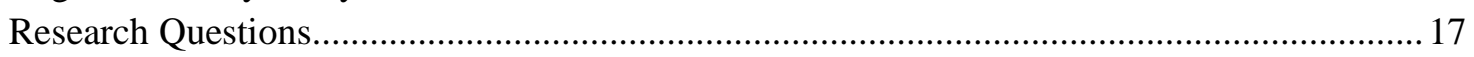

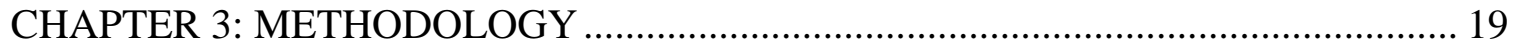

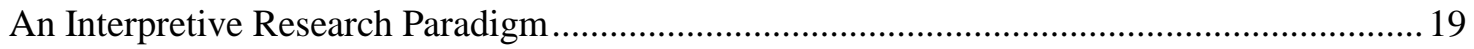

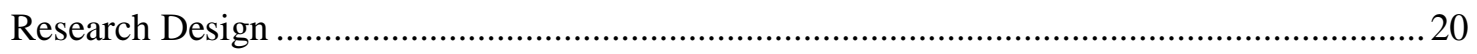

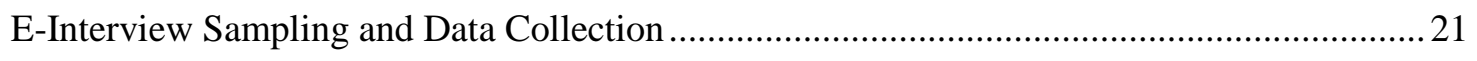

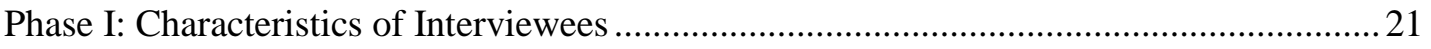

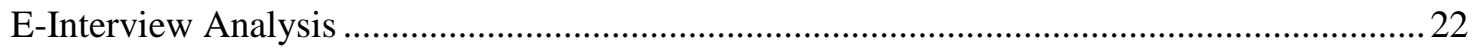

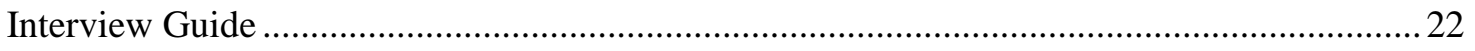

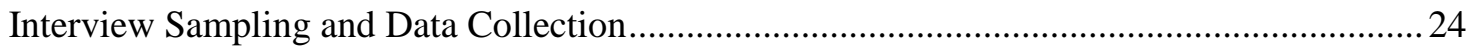

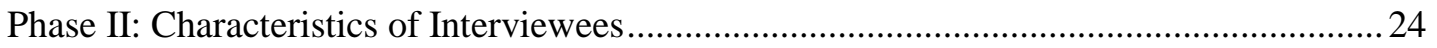

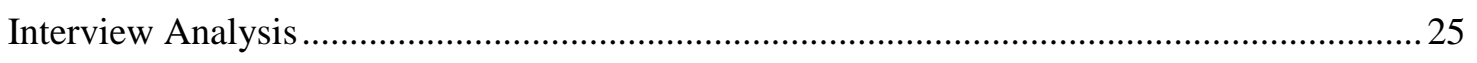

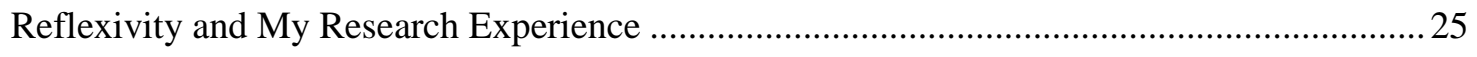

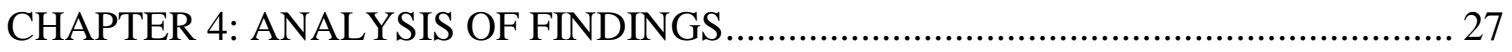

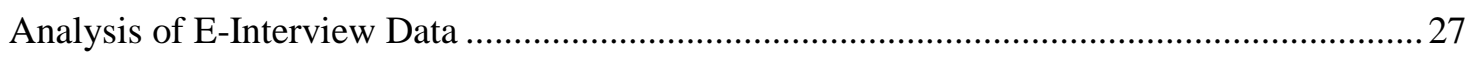

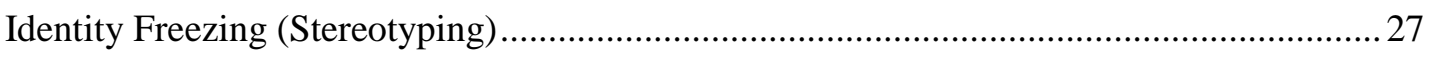

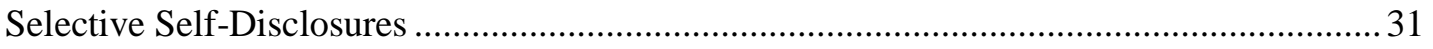

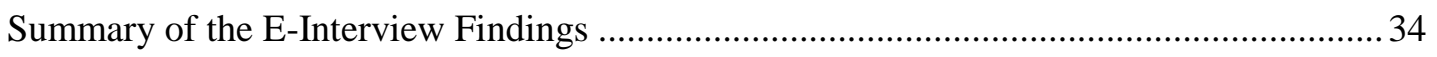

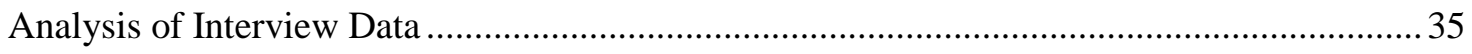

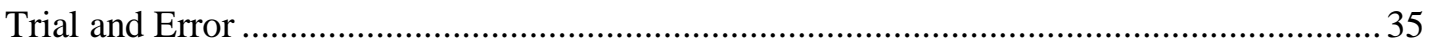

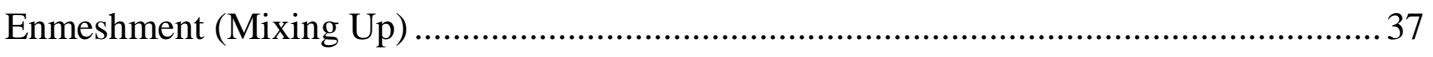

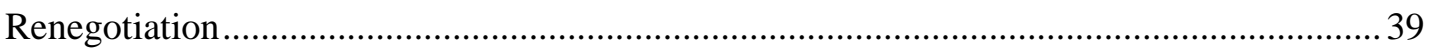

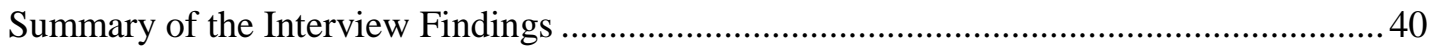

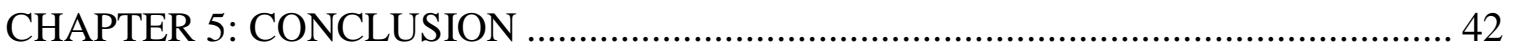

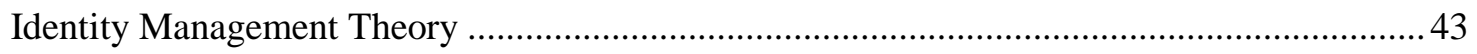

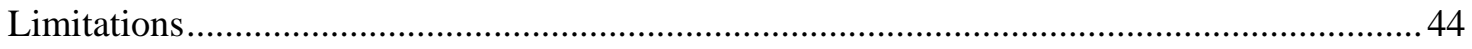

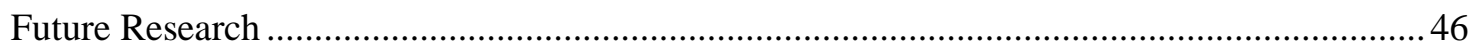

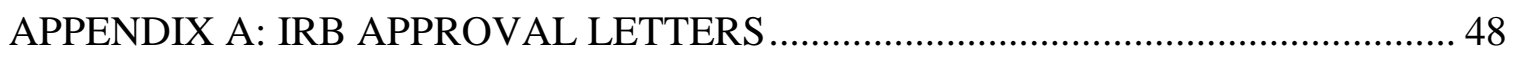

APPENDIX B: E-INTERVIEW QUESTIONS ........................................................ 51

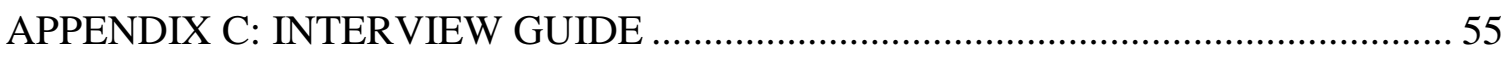

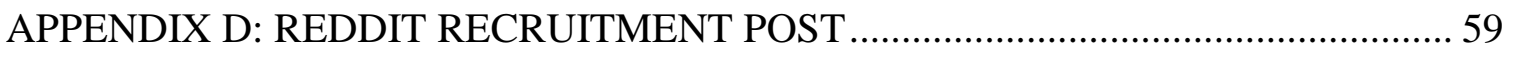

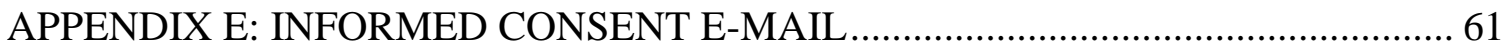


REFERENCES . 


\section{CHAPTER 1: INTRODUCTION}

The cultural focus of this study is polyamory as a non-normative relationship orientation. Culture is collectives of people sharing symbols of relating, identities, and social attitudes (Gudykunst, 2003). Shared attitudes of polyamorous people create a culture of meaning. Polyamorous culture not only shares common language to describe their relationships, but an understanding of how relationships should work. When interacting with monogamous individuals about relationship-related topics, intercultural communication across these social groups occurs. As polyamorous culture is distinguished from dominant monogamous cultural beliefs, polyamory represents a co-culture (Orbe, 1998). Intercultural communication involves face-to-face interactions between people of different cultures, including people of different nations, racial or ethnic groups, intergenerational interactions, and on (Gudykunst, TingToomey, \& Chua, 1988). As polyamory is a social identity set apart from one's national, racial, or ethnic identity, the type of intercultural communication examined in the present study is intergroup communication, or communication between members of different social groups (Gudykunst, 2003). Previous studies have examined social identities, like one's sexuality, as intercultural identities (Howard, 2012). I aim to illuminate how polyamorous people communicate their identities, and how they perceive intergroup communication regarding their relationship orientation.

Polyamory is a deviation from the monogamous social norm. The monogamous norms in North American society are reflected historically and endure in state laws. Historical origins situate women as men's property through marriage (Vogel, 1992), while women could be penalized for committing adultery. The justification of the laws assured genetic offspring for men. In 2016, 17 states still uphold adultery as a fineable offense (Rhode, 2016). While 
polyamory is a consensual form of non-monogamy, the belief pervades that a monogamous dyad is the only acceptable relationship arrangement. Polyamory is operationalized as a relationship in which each partner may have additional sexual or nonsexual romantic partners, while all partners consent to and negotiate the relationship arrangement. Polyamory is a type of consensual nonmonogamy which emphasizes multiple loving emotional relationships. The recent increase in popular culture's interest in polyamorous identities can be seen in new and traditional media treatments of this relationship orientation. Showtime aired a reality show about polyamory that broadcast for two seasons. Buzzfeed's "Ask a Polyamorous Person” video has over a million views (Buzzfeed, 2014). Even the Fox Network's television program New Girl casually featured polyamorous neighbors of the main character. Although the visibility and interest in the culture of polyamory appears to be increasing, there is little social science research examining polyamorous intercultural communication. The following report investigates people who identify as polyamorous, polyamory as a cultural identity, and how these individuals manage their identities.

The guiding theoretical framework for the study is identity management theory, or IMT (Imahori \& Cupach, 1993; 2005), which focuses on interpersonal communication and identity management between people from different cultures. The theory has been applied to intercultural interactions between people of different national backgrounds and different abilities, but not yet to people of different relationship orientations. Utilizing IMT to look at polyamory as a cultural identity highlights the intercultural negotiations for individuals situated in a monoganormative society. Further, I aim to illuminate how people who are polyamorous encounter stigma in regard to their identity. The inquiry focuses on polyamorous individuals as they interact with 
monogamous social landscapes, develop relationship identities, and negotiate differing cultural perspectives.

The literature review will examine monogamies and a variety of consensual and nonconsensual non-monogamous relationships that are distinguished from polyamory specifically. Additionally, this study contextualizes polyamory within a heteronormative and monoganormative culture in which heterosexuality and monogamy are privileged relationship models. Cultural privileging of monogamy creates the key conditions for the stigmatization of alternative relationship orientations such as polyamory. Below, Goffman's concept of stigma is examined with an eye on the possible ways polyamory can embody stigmatization within a heteronormative society. Though social understanding of polyamory is growing, common perceptions still focus on sexual practices, frame the individuals as nymphomaniacs, cheaters, or polygamists (Conley, Moors, Matsick, \& Ziegler, 2013). The actuality of polyamory is that the practice places importance on loving relationships and friendship rather than sexual exploration. Subsequent review supports the concepts of polyamory and social identity.

There is a significant gap in communication literature regarding polyamorous people, especially the nature of intergroup communication regarding polyamorous relationship orientation. Polyamory as a topic of study offers an abundance of novel communication-related research, spanning intercultural, interpersonal, relational, and health communication factions. Also, carrying out an empirically grounded research process to understand those that are stigmatized, my aim is to better understand the polyamorous identity and polyamorous individuals' interpretation of stigma. Further, the inquiry into polyamorous individuals' interpretation of stigma has implications for deeper intercultural understanding and acceptance of non-monogamous relationship orientations. 
In this chapter I have described the main concepts guiding my study and framed the rationale for research in the areas of polyamorous relational identities and intercultural communication. Chapter two contains a review of the literature, including the literature on identity management theory, polyamorous relationships, and stigma. Chapter three contains the research design and methods of data analysis. Chapter four will present the analysis of findings. Chapter five will present the conclusion of the findings, identity management theory, future research, and limitations of the study. 


\section{CHAPTER 2: LITERATURE REVIEW}

The following review of the literature covers the topics of identity management theory, polyamory as an intercultural identity, types of relationship orientations, and stigma. Following the literature review is the study's purpose and guiding questions. Next, in order to contextualize the topic of non-monogamy, the tradition of monogamy within the heteronormative society must be explored. Additionally, consensual non-monogamy as a concept will be defined, and the types of consensual non-monogamies will be detailed. With specific focus on polyamory as a relationship orientation, I drill down into the existing literature and its discussion of polyamorous identity. I aim to link the topics of identity management theory, the polyamorous identity, and the stigmatized identity.

\section{Identity Management Theory}

The purpose of identity management theory is to provide an explanation for the ways people manage their cultural and relational identities. The management of these identities is a fundamental concept in intercultural communication and intercultural competence (Cupach \& Imahori, 1993; Brown \& Levinson, 1978). Developed by Imahori and Cupach (1993), the theory encompasses communication across cultural situations and facework that occurs between cultural actors and their interpretations of interactions. The theorists explain how one's self-image, or identity, is performed during interactions with another individual. Imahori and Cupach built identity management theory from the foundations of Goffman's (1967) facework theories. Goffman's examination of face-to-face social encounters highlights the underpinnings of symbolic interactionism in both facework and identity management. What this means is the way we act towards people reflects how we view them and their social identities, and the way we 
conduct ourselves in those interactions reflects how we view our enacted identity in the social context.

Facework and competence are distinct features of identity management theory. In regard to cultural and relational identities, competence in this context involves the ability "to successfully negotiate mutually acceptable identities in interaction" (Cupach \& Imahori, 1993, p. 118). Competence requires that individuals in interpersonal relationships support one another's expression of identity. Face in this context provides the means by which we communicate our cultural and relational identities. In social interactions, we support one another's face and we do this by cooperative means (Goffman, 1967). Individuals possess two types of face desires positive and negative face (Brown \& Levinson, 1978). Positive face is the desire to be liked and accepted. To support one's positive face is to demonstrate approval of their attributes, physical appearance, accomplishments, or personality. Negative face references the desire for autonomy. To support one's negative face is to be tactful in interactions, and to avoid intrusion or constraint. A face threat involves an action which compromises another person's negative or positive face needs, and to undermine their identity (Brown \& Levinson, 1978). Face threats are common in the development and transitions between phases of identity management theory.

According to Cupach and Imahori (1993), the transitional phases outlined in identity management theory are necessary to develop intercultural relationships. The first phase is called Trial and Error. Within this phase the individuals from different cultures will look for similarities and common aspects of identity among one another. The cultural actors will also engage in identity freezing, or stereotyping one another based on preconceived information about each other's cultures. In the context of monogamous and polyamorous interactions, this typically occurs when the monogamous cultural actor threatens the positive face of the polyamorous 
cultural actor. Next, the Mixing Up or Enmeshment phase occurs, which is characterized by a mutual understanding of the relational identity, and downplaying differences between each other. Threats to positive face, or what is called the nonsupport problematic, occur often in this phase, which is characterized by cultural actors ignoring each other's cultural identities. The third phase is Renegotiation, which happens when individuals have achieved a distinctive cultural identity and mutual acceptance in interpersonally understanding each other's cultures. Within the context of the present study, this would require monogamous and polyamorous cultural actors to validate both relationship orientations and support one another's positive face.

There have been a variety of intercultural applications of identity management theory, though no other scholars so far have ventured to apply the theory to polyamorous individuals. Imahori has applied the theory to interethnic relationships $(1999 ; 2001 ; 2002 ; 2003)$ and Lee (2006; 2008) applied the theory to intercultural friendship development. Others have utilized the theory for managing relationships between individuals with and without disabilities (Merrigan, 2000). IMT demonstrates how people from different cultural identities can manage face-threats implicit in their interactions, and move forward with enmeshed relational identities, and further renegotiate separate cultural identities. Within this context, the theory can be applied to interactions between people of different cultures of ethnicity, relationship orientation, ability, or sexuality. Although identities like sexuality or relationship orientation are mostly invisible, interactions and tensions that arise about relationship-related topics are very common in a variety of contexts, including the workplace, doctor's office, and among friends and family. Cupach and Imahori (1993) argue that renegotiation of cultural identities allows the development of cultural competence and management of tensions and face needs. Face threats for polyamorous people may include being stared at if one is out socially with more than one partner, stereotyping, and 
burden of educating monogamous people about their relationship identity. The present study utilizes the definition of identity as developed by Collier and Thomas (1988). Intensity, scope, and salience comprise the three dimensions within the concept of identity. One individual can inhabit many identities (e.g. polyamorous person, mother, and best friend can all be identities of one person), and the social context determines which identity comes forward (e.g. the mother identity is enacted in interactions with one's children). The intensity of one's identity is related to how opening and overtly one can perform an aspect of themselves in a social situation. The scope of identity is related to the size of the group of people who share a common identity. For example, a relational identity has a small scope limited only to the people within a relationship. The concept of identity salience is one's feeling of importance and self-expression in a given social setting as an aspect of one's identity comes forward. Salience of an aspect of an identity changes depending on the social context.

\section{$\underline{\text { Heteronormativity and Monogamy as Cultural Standards }}$}

In North American cultural standards, the ideal sexual orientation is heterosexuality. The resulting heteronormative society places heterosexuality and monogamy at the top of the relational hierarchy, while all others are viewed as other, alternative, or deviant (Farvid \& Braun, 2013). The slippery slope arguments against same-sex marriage often loop in non-monogamy as they include reference to the loosening of moral codes, multiple partner families, and relational chaos (Sheff, 2005; 2006; 2011). Monogamy as a hegemonic tradition upholds norms of morality and how to conduct oneself in a relationship correctly (Anderson, 2010; Farvid \& Braun, 2013). Mono-normativity, or the norm of monogamy as default or normal, is a pervasive expectation that a dyadic relationship must be sexually exclusive. The concept of classical monogamy is 
defined as one man and one woman marrying as young virgins, maintaining a sexual relationship with only each other, and remaining sexually loyal until one or both people die (Goldfeder \& Sheff, 2013). With no-fault divorce providing dissolutions of marriages without question in 1984, classical monogamy shifted to serial monogamy, a relationship orientation wherein people seriously date or marry one person, stay sexually monogamous for a long-term period with one person, break up or divorce, and repeat the process over a lifetime (Robinson, 2013). Although the types of monogamy emphasize the dyad, the following section elaborates on the multiple partner models of consensual non-monogamies.

\section{Consensual Non-Monogamies}

The literature available on non-monogamy spans critical and celebratory examinations (Barker \& Langdridge, 2010), and is often not discussed without positioning non-monogamy in opposition of monogamy. While acknowledging the monoganormative society, the current study aims to present the types of non-monogamy as standalone relationship orientations with each having its own unique features. Types of consensual non-monogamies include polygamy, open relationships, swinging, and monogamish relationships. Additionally, polyamory as a type of consensual non-monogamy has its own nomenclature definitions including poly fidelity, and relationship anarchy.

Consensual Non-Monogamies (or CNM's per Conley, Moors, Matsick, \& Ziegler, 2013) allow for romantic and/or sexual relationships to develop within or beyond a dyadic union, and emphasize negotiation between all partners. Another umbrella term for CNM's is open, or open relationships. An open relationship is one that has a primary dyad at the center who are married or long-term committed, and the dyad may take on secondary romantic and/or sexual 
relationships. The umbrella term of open relationships includes those that are swinging or monogamish, as well as many polyamorous relationships, but does not include people who practice relationship anarchy (Sheff, 2013), which involves the rejection of hierarchical relationships, either in polyamorous relationships or dyadic relationships, and the idea that sexual or romantic relationships should not be privileged over non-romantic or non-sexual relationships (Barker \& Langdridge, 2010). Relationship anarchy allows for all relationships to evolve naturally without limits, and highlights love as an unlimited resource. Swinging involves the consensual swapping of otherwise monogamous romantic partners for sexual encounters (Sheff, 2013). Monogamish is a primarily monogamous relationship but includes occasional and negotiated sexual contact with others. Monogamish relationship rules may allow only one-night stands, or locational limitations like when either partner is traveling. Additionally, a longexisting tradition of CNM is the marriage type polygamy, which includes more than two partners. Polygyny is the most common form of polygamy, which is a marriage of one man and multiple sexually-exclusive wives. Polyandry is a marriage of one woman marrying multiple husbands, though it is much less common.

Polyamory as a central concept in this manuscript will be examined further in depth in the next section, and its distinction as a CNM focuses on romantic partnerships sets it apart. Further, polyamory does not model itself after monogamy, in that a dyad does not need to be at the fulcrum of the relationship (Barker, 2005). Triads and quads exist in which all relationship partners are dating one another, and there is equality, communication, and affection among all partners. Other relationships may call themselves a "V," in which one person is dating two (or more) people, but those people are not dating each other. The partners in the relationship that are not dating are known as metamours, or their partner's partner (Sheff, 2013). Poly fidelity is the 
concept that all of the partners in the relationship are committed to each other and the intimate group is a closed group sexually and emotionally (Barker, 2005).

\section{What is Polyamory?}

The literature attempting to define polyamory reveals the fundamental guidelines regarding what it means to be a partner in a polyamorous relationship. Partnership in polyamory, like other close relationships, primarily involves negotiation, sexual ethics, and intimacy. The concept of polyamory has been discussed in various literature describing it as multiple partners who are committed, romantic, and consensually non-monogamous (Barker \& Langdridge, 2010; Duma, 2009; Haritaworn et. al., 2006; Klesse \& Easton, 2006; McLean, 2004; Mitchell, Bartholomew, \& Cobb, 2014; Ritchie \& Barker, 2006). Polyamory is set apart from swinging and casual sex. In contrast to swinging, polyamory involves multiple relationship partners whereas swinging involves only multiple sexual partners. In polyamorous relationships, an emphasis on the importance of communication emerges consistently. Klesse (2006) conducted qualitative interviews and focus groups with those who identify as polyamorous and queer. The author's findings carve out the major themes of polyamory and the discourse on non-monogamy. Further, gender differences can shape the closeness of the relationships. Some female interviewees in Klesse's study felt their partnerships with women were more intimate than those with men, which is confirmed by research in gendered patterns of friendship and disclosure. Negotiation is another important element of polyamory - each individual person negotiates the direction and closeness of their relations with another. Honesty and consensus are important themes that shape the discourse of polyamory. 
Though the general tenets of polyamory can be parsed out, there is still debate about the language used to describe the relationship orientation. In using the category "relationship orientation," polyamory is semi-analogous to types of sexual orientation (Ve Ard \& Veaux, 2005). Klesse (2006) brings up the issue of representation, and how it hampers the forward movement of polyamory as a wider known practice. Klesse argues that pluralistic sexual ethics are difficult to achieve if those involved cannot agree on rules and definitions of their practice. Ritchie and Barker (2006) discuss how people in the polyamorous community create language to describe their experiences and construct a collective understanding. Social identities emerge as collectives create new words to describe them (Ritchie \& Barker, 2006). In their study, Ritchie and Barker examine online web discussion groups for origins and tracking of new words in the polyamorous community. Further, the telling of stories of polyamorous people cannot be accomplished unless new words are created - words such as infidelity, promiscuity, adultery, and cheating are the only words that many people can correlate when they are first learning of polyamory.

\section{$\underline{\text { Polyamory as a Co-Culture }}$}

Culture is any collective of people sharing symbols of relating, identities, and social attitudes (Gudykunst, 2003). Co-cultures exist within a dominant culture, often sharing many of the dominant cultures' norms, attitudes, and behaviors but also differentiate themselves from the dominant culture by developing unique beliefs and behaviors (Orbe, 1998). The shared attitudes of polyamorous people create a culture of meaning through common language to describe their relationships and attitudes about relationships and love. As a co-culture, people in the polyamorous community often adapt dominant cultural norms and values and give them new 
meaning. In dominant North American culture, commitment often means both the intention to maintain a relationship with monogamy as an implied strategy for relationship maintenance. For example, although hierarchical polyamorous people do not equate commitment with sexual and romantic exclusivity, they still value commitment and use it to distinguish themselves from other CNM co-cultures such as swingers and relationship anarchists.

Being polyamorous is both an individual identity and a social identity, in that a person can embody the identity but also find social identification with other polyamorous people. Another social identity set apart from one's national, racial, or ethnic identity is the LGBTQ+ or queer identity. LGBTQ+ stands for lesbian, gay, bisexual, transgender, queer, and more, and queer is an umbrella term that covers anyone who is outside of normative gender or sexuality. Although the LGBTQ+ culture varies across geographic locations, common elements for shared cultural identification symbolize community. These common elements in queer cultural identification include words, social habits, art, and geographic location. The same common elements are reflected in polyamorous culture. Similarly, both cultures often share "coming out" narratives (Barker, 2005). Additionally, Cultures develop their own language to describe themselves. Polyamorous individuals have a vocabulary to describe their multiple partners, including terms for their partner's partner, like "metamour” (Richie \& Barker, 2006). Additionally, compersion is a term coined by the polyamorous community which describes the feeling of happiness one feels for their partner when their partner is happy with another person (Duma, 2009). As cultures develop, deeper understandings are shared between members and identities strengthen with collective meaning. 


\section{$\underline{\text { Non-Consensual Non-Monogamy }}$}

The primary differences between polyamory and cheating within a monogamous relationship is the consensual nature of the polyamorous relationship orientation and the communication between all parties involved. People who practice non-monogamy without consensual terms cite various reasons for engaging in these concurrent romantic situations. As most outsiders will view non-consensual non-monogamy as cheating, individuals engaged in the relationships describe various reasons for extra-dyadic affairs (LaSala, 2004). LaSala (2004) describes affairs in terms of physical need fulfillment and sexual exploration, while emotionally the partners are monogamous. It is important to emphasize that while non-consensual nonmonogamies are common, they are not the focus in this study. Mint (2004) describes the main differences between consensual and non-consensual non-monogamy, and how the scripts do not

exist to describe consensual non-monogamy yet. The author clarifies this misunderstanding - the opposite of monogamy is not non-monogamy, but cheating. Mint (2004) is highlighting the contrasting ideas of cheating versus fidelity as a power struggle, and in examining these ideas one can reconstruct polyamory as a solution outside the system. Social power is exercised through the locus of monogamy, and cultural ideas of cheating and jealousy enforce the dominance of monogamy. What this means is if we continue to look at other relationship orientations through the lens of monogamy, anything that deviates from it will not be accepted. Next, I will delve deeper into polyamory as a stigmatized identity.

\section{$\underline{\text { Stigma and Polyamory }}$}

Here I am shifting from discussing non-monogamy and polyamory as concepts to looking at polyamorous people as a population within a heteronormative and monoganormative society. 
With a comprehensive unpacking of Goffman's Stigma (1963), the present study examines how this concept applies to non-normative relationships. According to Goffman (1963), when one is identified with a mark of dishonor, they are considered stigmatized; that is, marking an individual as unlike us in a profoundly wrong way, and casting a negative light to this differentiation of the self compared to another (Crocker, Major, \& Steele, 1998). As polyamory deviates from the monogamous cultural norm and is widely stereotyped as sexually deviant or selfish, stigma is attached to their relationships. Stigmatized individuals are characterized as weak, dishonest, or full of unnatural passions (Goffman, 1963), and polyamorous people face selfish, immature, or perverse characterization. Goffman's theories on stigma outline who disqualifies from social acceptance and why nonconformity produces this result. Further, Goffman defines three types of stigma: physical disfigurements, moral failings, and "tribal stigma" (referencing race, religion, and ethnicity). The theory contains individual psychological elements and social components that contextualize the phenomena (Smith, 2007). The psychological component is the identity, and once affected by stigma, it is the spoiled identity. Enacted stigma is characterized by interpersonal discrimination, rejection, devaluation, and social avoidance (Link \& Phelan, 2001). In this study, I examine these repercussions for polyamorous people and the larger role of risk and power in being out as polyamorous.

Social stigma is perpetuated by society, but more specifically and globally, by those with power and privilege. People at the top of social structures with resources set and maintain social rules. As Anderson (2010) describes, polyamory is viewed as more stigmatized than cheating despite the lying and violation of relationship agreements, cheating at least implies an attempt at monogamy, however superficial. Within this context, monogamy is a reflection of one's morality, anything that deviates from this norm, such as polyamory, is stigmatized as immoral. 
Further investigations of stigma and power in regard to polyamorous identity aim to uncover how power influences one's comfort and ability to be open about their identities. In the workplace, for example, would be one area in which someone may not be able to disclose their relationship orientation. According to Emens (2004), there are no laws prohibiting discrimination of relationship orientations, which means polyamorous people have no legal rights to fight back if they are fired or marginalized in the workplace.

Research on polyamorous communities demonstrates that job loss and estrangement from family members are some of the consequences for non-monogamous individuals revealing their identities (Sheff, 2005). Monogamous people are rated more favorable than non-monogamous people, and the belief that monogamy is more moral and less risky than non-monogamy perpetuates stigma of the relationship orientation (Anderson, 2010; Conley, Moors, Matsick, \& Ziegler, 2013). Rambukkana (2004) describes the relationship orientation as often perceived as a bridge between normative monogamy and a culture of radical sex. The author describes the experience of creating a polyamory club on a college campus, and how the negative reactions were often coming from a defensive, uninformed place. Perpetuation of stigma regarding nonmonogamy can come from unawareness, but often there are much larger structures in place. The nature of monogamy is tied to the control of female bodies and capitalist structure in Western society (Anderson et al., 2010). Within this idea, as autonomy of individuals increases, as does social disruption and chaos.

The present study aims to explore the concept of stigma as it exists in monoganormative social landscapes. Polyamorous individuals may evade disclosing their relationship orientation to avoid stigma. Goffman (1963) requires for those who are stigmatized to be aware of their stigma, as well as others to be aware of it. Being socially stigmatized is to be discredited and humiliated, 
and to be privately stigmatized is discreditable. Goffman describes passing as a management behavior used to conceal a stigmatized identity. The behavior is characterized by evading the topic of relationship orientation, or concealing multiple partners and a discreditable identity. Within this context, Young (2014) posited that polyamorous people attempt to pass by acting as monogamous or single in social situations. Being polyamorous is typically not a visible identity in day to day interactions, thus the stigmatized identity emerges when the individual discloses their relationship orientation, or it is uncovered.

\section{$\underline{\text { Research Questions }}$}

This inquiry was guided by the following three research questions: How do individuals communicate about the development of their polyamorous identity within a monoganormative society? What types of identity management tactics do polyamorous individuals utilize to navigate a society in which monogamy is the norm? And finally, what part does stigma play in the communication between polyamorous and monogamous individuals?

The research questions were derived from an interest in non-normative relationships, identity management, and an interest in the meaning of a stigmatized relationship. The first question highlights the communication and identity management practices of the community. In discussing their relationships, do polyamorous people make jokes, or evade talk about their relationship orientation? Is there an effort to de-emphasize the differences between themselves and monogamous individuals? The second and third questions aims to uncover how polyamorous individuals experience stigma - from friends, co-workers, the media, or in the dating scene. In discussing their relationships, do they experience stereotyping and or hostility? Further, I aim to 
uncover if a power dynamic exists among those that stigmatize polyamorous relationships and polyamorous people.

The intent of this study is to demonstrate how polyamorous individuals manage their identity in interpersonal interactions within our monoganormative society using identity management theory as a framework. While previous research has surveyed polyamorous individuals about their intimacy, satisfaction, or self-perceptions, there is little existing literature on polyamorous people and their experiences of intercultural communication. The rationale for using qualitative methods is to explore a population and their emotions, narratives, and lexical choices in describing their relational identities. The e-interview was an exploratory open-ended questionnaire administered via online polyamorous communities. The study that followed was one-on-one interviews via phone call. 


\title{
CHAPTER 3: METHODOLOGY
}

\author{
$\underline{\text { An Interpretive Research Paradigm }}$
}

This study is an exploratory investigation into polyamory and identity, and involved gathering and interpreting qualitative data. Using qualitative methods allows one to understand complex issues with rich description. Methods of e-interviews and phone interviews grant access to witness struggles and concerns from the polyamorous community. Individual relationships are complicated and diverse, though the shared findings that are common become meaningful through interpretation. In this way, the study is situated in the interpretive paradigm and assumes a social constructionist approach. Though the polyamorous social culture is a collective construction of common language and meanings, I recognize the subjectivity of all the interviewees in the study and do not claim to represent all polyamorous communities.

The first phase of the study, which utilized e-interviews, was IRB approved by University of Central Florida and included questions for another study regarding polyamorous communication. The instrumentation in the e-interviews were selected to elicit unique responses, but also due to the exploratory nature of the research. Three instruments were used throughout the study. The first measure inquires about demographic data from interviewees, in both the einterviews and the phone interviews. Second, e-interview questions asked interviewees about their experiences of stigma and self-disclosures. Finally, the 22 one-on-one interviews (via phone call or Skype) provide depth of data and understanding of the topic from the interviewee's point of view. The data from the e-interviews are a foundation for the second phase of the study, and the interviews are the primary data used to answer research questions.

Additionally, this report acknowledges my position as a processor of data interpretation. I am a scholar of communication and a feminist, and thus curiosities in relationships and identity 
are deeply rooted in my interests, both academic and personal. I aim to examine the variety of ways relationships can manifest outside normative boundaries, and to explore the diversity of relational identities that exist beyond the dominant monogamous culture. Personal reflexivity involves reflecting upon the ways my own bias, beliefs, and social identities shape and influence the research. These methods allow me to become an instrument of interpretation of the data, but also the data have power to change me as a researcher.

\section{$\underline{\text { Research Design }}$}

As qualitative research composes many voices in a population to explore a topic deeper, the answers to the guiding questions cannot be easily explained. Creswell and Creswell (2007) describe qualitative inquiry as an "intricate fabric composed of minute threads, many colors, different textures and various blends of material" (p. 42). The goal of the study is to gain insight into a little studied population and to generate discussion regarding consensual non-monogamy, and to examine the identities of polyamorous individuals as they interact in a world set up for and by monogamous people. The two phases of the study - the e-interviews and the phone or Skype interviews allow for interviewees to have their voices heard. Previous studies on polyamorous identities have used e-interviews to answer questions regarding relationship identity formation, in which interviewees typed their responses. The method is justified as much of the negotiation for rules and meanings of polyamory occur on the Internet (Barker, 2005). By utilizing a medium that members of the community already trust, such as the Polyamory and Non-Monogamy subReddit pages on Reddit.com, my goal was to recruit individuals who are comfortable discussing their relationships while also meeting community members where they feel safe already. 


\section{E-Interview Sampling and Data Collection}

Online discussion forums have become a growing source of data collection in qualitative research. The forums provide unique opportunities for researchers to observe, request access, examine lexical choices, and recruit interviewees (Im \& Chee, 2012). The sample was a strategic and purposive convenience sample acquired from online forums. Interviewees were people who voluntarily took the e-interviews which was promoted in a post on the online social network Reddit. Although recruitment via online communities for e-interviews has inherent limitations, the method provides a national approach for sampling an adequate number of individuals who are polyamorous (Im \& Chee, 2012). The recruitment posting on Reddit.com and the front page of the e-interviews stated that interviewees must be over the age of 18 , not incarcerated, and identify as polyamorous. The recruitment post stated the e-interviews would take between 25 minutes and 1 hour of interviewee's time.

This sample was strategic as these active online forums have many interviewees who post often about their relationships and identities as polyamorous individuals. The open nature of the group promotes sharing with relative anonymity, and so this group would likely be receptive to writing about their relationships via e-interviews.

Phase I: Characteristics of Interviewees

The demographic information collected for the e-interviews included the gender of the interviewees, age, sexual orientation, and racial background. Thirty-nine interviewees completed the e-interviews. Twenty-four identified themselves as women, fourteen men, and one agender individual. Thirty-three were White/Caucasian, one Hispanic/Latino, one Indian, two multi- 
racial, and two selected other. Regarding sexual orientation, there were twelve straight individuals, seventeen bisexuals, two pansexuals, five queer individuals, one "hetero-flexible" individual, and one selected other. The age range was 21-41 years old, and the average age was 28.5 years old.

\section{$\underline{\text { E-Interview Analysis }}$}

The e-interviews data was analyzed using methods of thematic analysis (Owen, 1984). Owen's framework of analysis involves examination of discourse. The e-interview data was downloaded into an Excel spreadsheet from Qualtrics and cleaned for empty cells. The first round of analysis involved close reading, open coding, and assembling of themes for several rounds. Following Owen's guidelines for thematic analysis, during the close reading I coded the e-interviews for recurrence and repetition as the themes came up again and again. Forceful and unique segments were coded as well. As the e-interviews are typed and not spoken, use of all caps, exclamation points, or unusual wording provided significance in this criterion. Theme assembly followed with a Word document with headings for each major theme organized the data. Color codes for each theme and sub-theme visually captured the significant findings. Comment flags on each data signified my thoughts and deeper analysis as they answered the research questions. The exploratory nature of the analysis allowed for fluid emergence of themes from the body of data.

\section{Interview Guide}

IRB approval was obtained for an amended study to include phone interviews. For the one-on-one interviews via phone call or Skype call, interviewees were again recruited via the 
public forum subReddits, Polyamory and Non-Monogamy. Online means of recruitment was used as the internet has been a central place of communication, education, and cultural development for many polyamorous people, and has contributed to the growth of polyamorous communities (Barker, 2005). A Qualtrics form was linked from the recruitment post, which elicited interviewee's e-mail, phone number, and name they'd like to be called. I followed responses with an e-mail detailing the purpose of the study and their informed consent information regarding their rights as an interviewee, how I will keep the data safe and secure, and consent for recording the interviews. I answered questions on the forum post regarding my research purpose for clarification and to make recruits feel comfortable proceeding with the study. Once interviewees responded to the informed consent e-mail, the phone interviews were scheduled. Interviews conducted over phone and Skype call were collected over three weeks, and a total of 22 interviews were collected, ranging from 20 minutes to 1 hour.

The interview guide was divided into three sections: introduction questions, polyamory and culture, and Retrospective Interview Technique, or RIT (Huston, Surra, Fitzgerald, \& Cate, 1981). RIT required interviewees to recall development, or turning points, of their polyamorous identity and interactions with monogamous others from the past to present day. All interviews were digitally recorded with the in-call app, Cogi. Hand-written notes about repeated themes and unique experiences were recorded during the interviews. Numbers were assigned to each interviewee in the cataloging of the data, and subsequently pseudonyms designated interviewees in the manuscript. Other identifying information was redacted to further remove the identity of the interviewees. 


\section{$\underline{\text { Interview Sampling and Data Collection }}$}

Interviews were conducted over three weeks in early 2016. Of 22 interviews, two were conducted over Skype calls and the rest occurred via phone call. Rapport was established via email during which interviewees were able to ask questions about the study and schedule a date, time, and medium of contact for the interview. At the time of the interview, rapport was further established by detailing the purpose of the study, and allowing for any questions before the conversation began. I explain that the interviewees could decline to answer or withdraw from the interview at any time.

I transcribed approximately 6 hours of the data, and paid for professional transcription via the service Rev. The transcriptions resulted in 330 single-spaced pages of transcribed data. After each transcription was completed, I listened to the digital recording 2 to 3 additional times while highlighting repeated themes or unique findings.

\section{Phase II: Characteristics of Interviewees}

The demographic information collected for the interviews included the gender of the interviewees, pronouns, age, sexual orientation, racial background, how many years they have identified as polyamorous, and their current relationship dynamics. Thirteen identified themselves as women, eight men, and one non-binary individual. Twenty were White/Caucasian, and two were racially mixed (half White and Chinese, and half White and Mexican). Regarding sexual orientation, there were eight "hetero-flexible" individuals, six pansexuals, four straight individuals, two bisexuals, one "homo-flexible" individual, and one that responded, "it's complicated." The age range was 23-49 years old, and the average age was 30.4 years old. 


\section{$\underline{\text { Interview Analysis }}$}

The steps of thematic analysis followed close reading, then coding for frequency and intensity (Owen, 1984). Using the phases from identity management theory as a guide, the patterns of the themes fit into the categories presented in the following results. The first round of analysis involved close reading, and coding for data that fits in one of the three categories of IMT. Forceful and unique utterances were coded as well. Theme assembly followed with a new document with headings for each phase of IMT. Color codes and comment flags on each data signified my interpretation. As the interviews produced many similar themes throughout the interviews, I believe data saturation occurred.

\section{$\underline{\text { Reflexivity and My Research Experience }}$}

There are ethical issues to consider regarding entering an online community with intent to research. I recognize that the subReddits Polyamory and Non-Monogamy are safe places for polyamorous and non-monogamous people to go for community, friendship, and conversations with like-minded people who share their relationship orientation identity. For the e-interviews, I asked the moderators of the subReddits for permission to post my link. For both studies, I wanted to make my intentions, the risks, and benefits as clear as possible with my post, and included the University of Central Florida's IRB contact information in the posting and the informed consent e-mail. I also answered any questions or comments on the posting that desired clarification or additional information about my research purpose. It was important to me to demonstrate goodwill to the communities, at least in a small way via these correspondences. I muted the recruitment postings when data collection concluded so they may not become embedded in the message boards. 
Despite my personal and professional interest in polyamory, I have not experienced a polyamorous relationship. This affects my ability to relate to interviewees in some ways. Although I have many close friendships with those who are non-monogamous, and I have read many texts regarding non-monogamy, written many papers, and have a deep, personal desire to promote and destigmatize non-monogamy as a relationship orientation, I cannot know by way of experience the stigma faced by those in non-monogamous relationships, or feeling happy that my partner is happy with another partner. Because of this position, I have been very thoughtful of my language choices and interpretations, and asked for clarification during interviews if I'm uncertain of a community-specific term. Throughout the research design, data collection, and analysis of the data I have tried to be rigorously reflexive about my biases.

In this chapter I have described the research design and methods, and justified my choice of qualitative investigation. I have also reflected upon my experience as a qualitative researcher. The upcoming chapters will present characteristics of interviewees, examples from the data, and discussion of the findings. 


\section{CHAPTER 4: ANALYSIS OF FINDINGS}

This chapter contains results from the e-interviews which provided a departure point for the subsequent phone interviews. Additionally, this chapter contains data from twenty-two interviews conducted via phone call. Contents of this chapter include interviewees' exemplars, and analysis of their communication regarding the phases of identity management as they developed. Data interpretation from the e-interviews and phone interviews reflect the themes that emerged.

\section{$\underline{\text { Analysis of E-Interview Data }}$}

The following are findings from the e-interview data. The exploratory questions focused on polyamorous individuals' experiences of stigma and disclosures of relationship orientation within the monoganormative society. The first most common theme that emerged from the study was Identity Freezing (Stereotyping), with sub-themes of Sexual Deviance, Selfishness, Less Commitment, and Unsustainability. The second most common theme was Selective SelfDisclosures, with sub-themes of Self-Censoring in Professional Settings, Factors that Influence Self-Disclosure, and Dealing with Monoganormative Reactions. Identity Freezing refers to reactions polyamorous people have received from monogamous individuals which are based on stereotypical information or images of their culture. The concept of Identity Freezing is derived from Cupach and Imahori’s (1993) Trial and Error phase of identity management.

\section{Identity Freezing (Stereotyping)}

Throughout the e-interviews, one of the most prevalent themes was Identity Freezing, or stereotyping. The title of the theme, informed by identity management theory, was added 
retrospectively after the open coding methods. When interviewees were asked about their experiences disclosing their polyamorous relationship orientation, nearly all interviewees experienced an individual responding to their disclosure of their relationship orientation with information that was offensive, generalized, or simply untrue.

\section{$\underline{\text { Sexual Deviance }}$}

Among these stereotypes, the focus on the sex lives of polyamorous individuals is a primary concern for many. Their concern often takes form of judgment, probing, and assumptions of group sex or partner swapping. The first sub-theme is called Sexual Deviance, as those whom receive these comments are looked upon as perverts. The sexual deviance theme highlights the curiosity and assumptions regarding the sexual experiences of polyamorous individuals. The understanding of outsiders is primarily focused on the aspect of multiple sexual partners. For instance, A., a 41-year-old straight man said, "I'm not offended by it, but people tend to be curious about our sexual arrangements." Further, close friends and relatives were said to make sexual generalizations about individuals' pursuit of multiple relationships. C., a 31-yearold bisexual woman, said her mother commented, "He only wants a threesome!" in regard to her boyfriend's approval of C. pursuing other women to date.

Other interviewees describe reactions of disgust focused on polyamorous sex lives. G., a 23-year-old queer agender person describes how their friends referenced another polyamorous person with two boyfriends. G. said, "The other friend asked why anyone would want to date someone like that. The first friend then told us [the polyamorous friend] had "the clap," and the second friend said "Ewwwww, so gross," laughed, and then said, "Well, she kinda deserved 
that."” Similarly, I., a 39-year-old heteroflexible woman, said she was told, “That's gross. Just have sex with your wife." The intense language in their examples is contrasted with the example from A., in which he describes experiencing personal probing about his sex life but is not offended by it. Throughout these examples varying in degrees of severity, the interviewee's relationships are diminished to their sex lives, which ignores their romantic-centered identity. The messages each of these interviewees receive are grounded in the monoganormative cultural paradigm of their everyday interactions. Internalization of monogamy as the moral grounding for sexual conduct leads to the interviewees being looked upon as motivated only by sexual interest. Given this moral positioning, living a polyamorous life becomes socially stigmatized.

\section{$\underline{\text { Selfishness }}$}

The theme of Selfishness was expressed again and again in similar responses. Many interviewees encountered confusion and rejection of the concept of polyamory, with acquaintances and friends casting the interviewees as inconsiderate partners interested only in their own relational satisfaction.

Interviewees gave examples of common responses. For instance, K., a 24-year-old bisexual woman said she heard, "Why isn't [partner] enough for you?” Similarly, M., a 26-yearold bisexual man said he was asked, "Isn't that just being selfish?" The interviewees were described as greedy, hedonistic, and perverse in their desire for more than one relationship. Stigma and marginalization charge the interviewees as inconsiderate partners. The monogamous others encountered by interviewees are working from a dominant moral belief that nonmonogamy is immoral, and find character flaws in people who do not ascribe to their beliefs. 


\section{$\underline{\text { Less Commitment }}$}

Similar to the theme of selfishness, the theme of Less Commitment is a response echoed throughout the data as it was experienced by the interviewees. O., a 31-year-old bisexual woman heard from friends and acquaintances that, "there is no commitment in poly." Likewise, N., a 24year-old straight woman, was told that, "I will always come second to my partner because he is married." The interviewees experienced invalidation of their commitment, relationship orientation, and relationship dynamic. In N.'s case, the polyamorous relationships outside of the married dyad are viewed by monogamous others as less important, careless hook-ups. Additionally, individuals were cast as detached and careless in their relationships. The monogamous others are responding to the polyamorous interviewees from a framework in which monogamy suggests commitment, and polyamory is likened to cheating.

\section{$\underline{\text { Unsustainability }}$}

The Unsustainability theme demonstrates the invalidation experienced by polyamorous individuals as they navigate interactions with monogamous people in various social landscapes. The theme of an unsustainable identity arose as polyamorous individuals reported from various sources they have been told that they will change, and their polyamory is a phase. Z., a 27-yearold straight woman, heard that she, "will change after [she] meets "the right guy."” Monogamous others dismiss the validity of Z.'s relationship orientation as a symptom of trial and error in dating, and project that polyamory is a temporary choice. F., a 24-year-old bisexual woman, experienced unsustainability in her polyamorous orientation being viewed as, "a sign of immaturity, to be outgrown." Not only does this response reflect affront to the polyamorous 
individual, but also a misunderstanding of polyamory as a relationship orientation. Similarly, B., a 28-year-old queer woman was told by an OB/GYN, "You can't do this forever," in regard to her polyamorous relationships. Not only was this interaction stigmatizing, but it reflects the cultural incompetence of the $\mathrm{OB} / \mathrm{GYN}$ in approaching polyamorous patients. As doctor-patient relations have inherent power dynamics, the authority of the doctor serves to further stigmatize the polyamorous individual.

\section{Selective Self-Disclosures}

Another issue related to identity management involves selective self-disclosure of personal information. In the present study, a selective self-disclosure is a context dependent disclosure of one's relationship orientation. The salient sub-themes in this area include Self Censoring in Professional Settings, Factors that Influence Self-Disclosure of Polyamory, and Dealing with Monoganormative Reactions. Because of the stigma associated with alternative relationship orientations that can result in physical violence, job loss, and other threats to both identity and life quality.

\section{$\underline{\text { Self-Censoring in Professional Settings }}$}

In the data, many individuals mention the workplace as a setting in which they do not self-disclose about their relationship orientation. As there is a power dynamic within the workplace (the employer controls your ability to be promoted, make money) and individuals spend many hours a week in these environments, the polyamorous individuals aim to protect themselves from professional threats. R., a 24-year-old bisexual woman, describes her concerns 
about disclosing her relationship orientation in the workplace, "In a work environment I feel more hesitant to disclose my relationship status based on the company policy regarding discrimination. I would hate to lose my job over 'moral' impasses with an employer or coworker." Frequently in their responses, the interviewees discuss how power in the workplace influences their choice to avoid disclosures. Power provides primary reinforcement of stigma and stigmatized identities in the workplace. Although people do not readily discuss sexuality in the workplace, the casual reference to multiple partners (as one might reference if they have one partner) creates constraint for polyamorous people. The workplace as a setting involves a different set of social rules, including desire to be perceived as trustworthy, competent, and in control of one's emotions and private lives. As the workplace is an area of heightened self censoring, interviewees will avoid positive face threats not just to protect their character, but to also self-preserve their jobs and income.

\section{Factors that Influence Self-Disclosure of Polyamory}

The polyamorous individuals list many factors that influence their self-disclosure of their relationship orientation. As they aim to protect themselves from stigma, they analyze the risks and benefits of their disclosures and if it will be welcomed, or if it's relevant. B., a 28-year-old queer woman, describes how she assesses self-disclosure of her relationship orientation - "Do I want to engage with this person in a way that requires it (e.g. potential new dating partner) and do I feel comfortable disclosing?" Mentions of comfort and safety from stigmatization surfaced frequently within this theme. In addition to feeling comfortable, M., a 26-year-old bisexual man, also wants to be in control of the disclosure. He said he will disclose, "if it feels like they are 
accepting of alternative lifestyle and wouldn't treat me differently. Or if I know they will find out anyway and I'd rather have them hear it from me." Most of the interviewees describe a fear of stigma and selective self-disclosure as a choice to be weighed to avoid stigmatization and discreditable identity. As relationship-related topics are common in most social scenarios, the interviewees may choose to evade questions or lie about their relationship orientation if they feel it is not safe or necessary to disclose. As the polyamorous relationship orientation is not a visible cultural identity, it is an individual choice to disclose. Once the identity is disclosed, the interviewees risk being discreditable and privately humiliated.

\section{Dealing with Monoganormative Reactions}

In this theme, the interviewees highlight the management tactics they utilize in Dealing with Monoganormative Reactions. The interviewees again are strategic in their self-disclosure of their identity, and concerned with responding to comments rooted in monoganormative cultural conceptualizations of relationships and sexuality. Z., a 27-year-old straight woman, cites her lexical choices as a strategy for dealing with monoganormative reactions, and discusses the tenets of polyamory as a romantic relationship orientation. She said, "I will only disclose my status if someone asks questions regarding my relationship status. I will answer truthfully and disclose what is my point of view about romantic relationships without mentioning the word polyamory. If the person is not reacting positively I will end the subject." Once the identity is disclosed, many interviewees describe their experiences of ending the subject, or polite disengagement. Similarly, H., a 37-year-old pansexual woman, aims to dispel myths or 
misunderstandings about polyamory, but avoids heated conflict. She accounted, "When I get a negative reaction, I usually start at confusion. Then depending on context I explain poly (friend of friend, person who seems curious) or disengage politely (metamour's boss's friend, person who seems angry)." Interviewees feel they have to defend polyamory but are often asked to do so in a monogamous construction of relationship reality. In efforts to protect themselves and or rectify any misinformation about the polyamorous identity, the interviewees choose their words carefully, clarify any stereotypes, or change the subject to avoid stigmatizing reactions.

\section{Summary of the E-Interview Findings}

The two major themes from the e-interviews, Identity Freezing and Selective SelfDisclosures, suggested that the everyday interactions with the dominant monogamous culture bring about major challenges for polyamorous individuals. Stigma and face threats are common experiences for the interviewees, and the ways in which they navigate relationships (friendships, workplace interactions, family, etc.) pose many questions regarding how they make and sustain them with monogamous people. From these findings, identity management theory appeared to be an appropriate framework for moving forward, as the fluid development of interpersonal relationships among those that are polyamorous and monogamous are very similar to those of different cultures of ethnicity, ability, religion, and on. The selective self-disclosure theme connected to IMT in a profound way, as cultural competence and facework are necessary to negotiate how we relate to one another. 


\section{$\underline{\text { Analysis of Interview Data }}$}

The following are findings from the interview data. Using both the e-interviews data and identity management theory as a departure for research questions and interview questions, the themes selected are derived from the IMT framework. Cupach and Imahori (1993) developed the themes, which they call phases, of identity management in intercultural interactions. The phases are Trial and Error, Enmeshment or Mixing Up, and Renegotiation. The sub-themes derived within each phase have been detailed within the interpretation to differentiate unique experiences of the polyamorous interviewees. Further, a common theme in the interactions between polyamorous individuals and monogamous individuals is the experience of being the stigmatized other, and this most commonly emerged in the Trial and Error phase of identity management theory.

\section{Trial and Error}

Within this phase of identity management, the identity freezing problematic comes up as stereotyping the polyamorous individual. Others demonstrate intense interest and curiosity in polyamory, which results in positive-negative face dialectic. Interviewees view the stereotyping as face threats, and as outlined by Cupach and Imahori (1993), and there is an attempt at balancing the negative and positive face threat dialectics. A theme within this phase is the understanding by the polyamorous interviewees that the monogamous interaction partners are "working with a different framework" to understand relationships. Monogamous interaction partners have not come to see or accept the polyamorous relationships, and thus have not moved on to the next phase of their cultural understanding of their polyamorous friends. 
According to X.I., a 28-year-old hetero-flexible woman who is married to a man, Q., and dating one other person, she describes the most common positive face threatening question she receives from monogamous others. She said, ““'Is Q. okay with it?” Is what always seems to come up I guess. Because they are trying to scope out with how it works and that ... that seems to be maybe it's because I'm perceived as X.I. who is married to Q., rather than being perceived as X.I. who knows a lot of people and relates to them in different ways... ... Or they're, they're framework that they're using to understand these relationships is a bit different. That is a question that I do only get from ... I think I only get from the, the, the ones who are less familiar with it. Less familiar with myself, with me." As these interactions commonly come up with others who do not know X.I. very well, their questions arise in the Trial and Error phase of interactions between polyamorous and monogamous cultural actors. The monogamous others asking the questions of X.I. have not acquired cultural competence yet to develop a relational identity and identify boundaries for face support and face threats.

Further, T.H., a 27-year-old mostly straight man who is married to a woman with one metamour, describes how other monogamous cultural actors are "polite" in their questions aim to balance the self-other face dialectic and the positive-negative face dialectic. He explained, “Those who do know have generally responded with, uh, honest and polite curiosity. I've had some people, uh, in a mature way say that they could never live that way but seeing as its harming no one... they see no reason to throw stones ... After that comes some, uh, basic questions as to, "Well, how does that work then?" Uh, the monogamous mindset is too, too ingrained into our culture that most people can't see, immediately envision any other concept." Cupach and Imahori (1993) distinguish that within the Trial and Error phase, cultural actors pursue mutual education regarding their identities to establish boundaries and deeper 
understandings of their identities. When T.H.'s friends ask him about how polyamory works, they are attempting to comprehend his relationship orientation despite their monogamous mindset. C.C.'s experience in the Trial and Error phase echoes T.H.'s, in that she encounters curiosity, but not without face threats. C.C., a 28-year-old hetero-flexible woman with two longterm partners, describes her interactions with monogamous others: "Sometimes people are really excited and like, "Wow, I've never heard of anything like that before. I have so many questions," and it's like this really good positive curiosity. Some people are a little bit weirded out. They're like, "Uh, I don't know if I could do that. That's really strange..." Some people are straight up offended and disgusted and shocked and just, "How could you do that? I can't believe that. Why would you do that?" It definitely runs the whole gambit." Stigma and identity freezing play a large role in the struggles to communicate the polyamorous identity. Much like the first phase of the study reflected, sexual deviance surfaces as an identity freezing stereotype during the Trial and Error phase. C.C. continues, "The other questions tend to be surrounding sex. Either it's like, well do you have group sex all the time? Which the answer to that is easy. It's no. ...I think that part of that question is not only kind of reassuring people, like yes, the majority of poly people are very responsible with their sexuality." As C.C. assures the monogamous others of responsible sexuality, she is attempting to not only work against stigma, but also establish common values of consensual and safe relationship practices.

\section{Enmeshment (Mixing Up)}

Within the Enmeshment phase of identity management, the interviewees placed other shared identities before their polyamorous identity, downplaying their differences. Polyamorous and monogamous interaction partners move from the Trial and Error phase once they establish 
commonality between them and converge symbols and rules for interpretive frameworks. While not entirely comfortable with cultural differences, the interaction partners will still attempt to resolve but not entirely eliminate face problematics and dialectics. A.B., a 27-year-old straight man who is married to a woman, describes his view of his polyamorous identity. He said, "Before we're polyamorous, we're people ... and that no matter where we are in in our life, that's merely a facet of our life. Um, [in] America today... we tend to see one facet of a person and assume that's all that they are. ... That's not the biggest thing about any polyamorous person I know. It's, it's just a part of our lives." A.B. is diminishing the polyamorous identity as merely a facet of his life. He is converging symbols of the everyperson, and places the polyamorous identity aside to be seen as a person.

Similarly, C.C. converges the polyamorous identity with "normal people" and "normal relationships.” She said, “These are normal people conducting normal relationships. They just happen to look a little bit different." With this statement, she aims for monogamous others to enmesh their interpretative frameworks for relationships to include polyamorous relationships. She continues, describing why this enmeshment is so important - "Looking at it kind of from more of a big picture sense, I think the most important thing is to divorce polyamory from... something that's deviant and degenerate, primarily because that's come up so many times in custody battles. There's a lot of poly parents who can't be out of the closet because they're terrified that their kids are going to get taken away from them... it's all too easy to kind of use that perception of polyamory as leverage in a court case." In describing the "big picture," C. C. is placing the parent identity first. Stigma is a barrier for parents to come out as polyamorous, but also a barrier to reaching renegotiation. The interviewees in these examples are demonstrating ways in which monogamous and polyamorous people can have commensurate systems of 
relation outside of their relationship orientations. While the exemplars fit the IMT Enmeshment phase requirements of moving toward establishing common desires, it does not reflect a development of shared relational identity. The data provides examples of one side of an intercultural interaction, and thus does not provide a complete view of the intercultural enmeshment with monogamous cultural actors.

\section{Renegotiation}

Within the Renegotiation phase, individuals are reflexive about their experiences being polyamorous within the monogamous culture and verify that their experiences can co-exist. While in the Enmeshment phase, cultural actors place importance on other facets of identity while aiming to establish common interpretive frameworks, the Renegotiation phase features a successful enmeshment and cultural actors mutually viewing their identities as assets. T.H., a $27-$ year-old mostly straight man who is married to a woman with one metamour, depicts the polyamorous identity as an asset and fulfilling feature of his relationships. He said, "It was seeing her have healthy relationships with five people all at once and all of them knew about, knew about each other and were okay proved to me that yes, my mind said, "Well, different from the massive, the massive culture, can work." ...because the same things will break up any relationship, not communicating to each other, not valuing each other." Again, an interviewee references the dominant framework, or massive culture, of monogamous others. The interviewee describes how monogamous and polyamorous relationships will encounter the same conflicts, regardless of the relationship arrangement.

Comparably, D. N., a 30-year-old hetero-flexible woman in three long-term relationships also describes her distinct relationship orientation as an asset. She said, "I'm very emotionally 
invested and I'm very deeply emotionally rewarded. And I'm not just like going crazy and (laughs) you know just sleeping with tons of people or something like that. Like I wish, but that's not the case. Um, yeah, I mean like honestly, most of us are really boring people who really like to do lots of nerdy things." She is dispelling the myths that there is more sex or sexually deviant behavior, and confirming the commitment that she wants as a polyamorous individual, just as monogamous people desire. A worldview in which polyamorous and monogamous people want the same things is the framework with which the interviewees are examining their relationships. The interaction partners are looking at themselves and their distinct cultural identities as an asset rather than a relational barrier. In this context, the polyamorous interviewees are highlighting the common desire for commitment between both monogamy and polyamory.

\section{Summary of the Interview Findings}

As identity management is applied to polyamorous individuals' accounts with monogamous people in their lives, there emerges distinct ways in which face threats serve to reinforce stigma, but also how cultural actors find common understandings. These identity management tactics provide many answers to the research questions. The Trial and Error phase contained the most variety of responses in regard to identity freezing, in that some polyamorous cultural actors had to balance the face dialectics and problematics, or the tensions in which they want to support their own face but also the other person's face. The Enmeshment phase exhibits the ways in which polyamorous people downplay their relationship identity in order to avoid questions, stigmatization, or to connect with people on topics other than polyamory. The Renegotiation phase demonstrates that different types of relationships can be viewed from the 
same framework, and the interviewees aim for interactional partners to view their relationship orientation as valid.

Lastly, there is no easy solution for destigmatizing polyamory and making these intercultural interactions conflict-free. After initial Trial and Error phases there is no way of knowing if the interaction partners (mainly on the monogamous side) acquired knowledge to successfully interact with polyamorous people in the future. Monogamous individuals relying on stereotypical knowledge about polyamory only serve to hurt interactions. Intercultural competence on behalf of monogamous individuals is needed for not only relational adaptation between interaction partners, but for wider social acceptance and understanding of polyamory as a relationship orientation. 


\section{CHAPTER 5: CONCLUSION}

The research questions that generated this thesis are, "How do individuals communicate about the development of their polyamorous identity within a monoganormative society?" "What types of identity management tactics do polyamorous individuals utilize to navigate a society in which monogamy is the norm?" And finally, "What part does stigma play in the communication between polyamorous and monogamous individuals?" This chapter begins by summarizing the convergence of the phases of the study, and the application of identity management theory. Limitations and future research will then be presented.

The areas in which the first and second phases of the study overlap highlight stigma as a significant restriction for intercultural communication between polyamorous and monogamous people. Although interviewees aimed to downplay differences between monogamous people and themselves, more often the interviewees felt as though they had to justify their relationships. Additionally, the e-interviews and the phone interviews both contained themes of sexual deviance or intrusive curiosity regarding the interviewees' sex lives. Although education among interpersonal relationships can serve to aid connection and dispel stereotypes, it leaves polyamorous people largely responsible for maintaining and addressing these potentially face threatening interactions. Stigma in the workplace served as another connection between the study phases, as the theme surfaced in the e-interviews and during phone interview data collection. Although it did not fit in the phase II theoretical coding, fear of stigma in the workplace surfaced in nearly all of the phone interviews. The fear of being harassed, othered, fired, and passed over for promotion were reasons some individuals gave for not disclosing their polyamorous identity in the workplace. 


\section{$\underline{\text { Identity Management Theory }}$}

Identity management theory brings forward understanding relationships of cultural difference, and the development of understanding identities different from our own. As insufficient cultural understanding can be rectified through communicative events, these relationships can be difficult to navigate. As polyamorous and monogamous people learn to embrace differences, and identify shared values, there can be a renegotiation of understanding between them, but not without addressing stigma and stereotyping along the way.

The first research question inquires specifically about how individuals communicate about the development of their polyamorous identity with monogamous others. While questions were asked of interviewees about how they came to identify as polyamorous, many responses did not include how these developments were perceived by monogamous interactional partners. Interview questions regarding disclosures and experiences of stigma were the primary source of data for the identity management phases.

This study could extend the literature on identity management theory as it is the first to apply it to relationship orientation or any intergroup communication. As the analysis of the einterviews data consistently reflected the polyamorous individual navigating reactions and interactions within the dominant monogamous culture, identity management theory supported an intercultural approach to communication between polyamorous and monogamous people. The research questions served to use IMT to uncover how polyamorous individuals' experience the conversations with monogamous people - whether they are friends, co-workers, family, the media, or in the dating scene. Additionally, the research questions probe the communication and identity management practices of the community. While the phases of identity management theory were successfully applied to the interview responses from interviewees, there was no 
transition of one phase to the next per interviewee. The themes of the individual phases were reflected in all of the interviews, but no one discussed how their relations with monogamous people developed from an Enmeshment phase where one would downplay their differences, to a Renegotiation phase where a common identity was mutually understood. As IMT is a dyadic theory, interviewing one polyamorous person and a friend of theirs who is monogamous may have produced results that reflect the entire story of one phase to another. This was a limitation of the study, but could potentially be a limitation of the theoretical application. As Cupach and Imahori (1993) did describe the phases as highly interdependent and cyclical (p. 203), the present study reflects the phases as somewhat fixed in position as the polyamorous communicators indicated they were often selective in their disclosures and word choices when describing their relationships. Not all of the interviewees expressed experiences of stigmatized or stereotyped identities, though this lack of fluidity between identity management phases could be due to the awareness of dominant monogamous norms.

\section{$\underline{\text { Limitations }}$}

Limitations of the study can be found in the sampling, and some features of the methodology. Although the findings of this study could serve to benefit the polyamorous community and their relationships with monogamous people, it is unclear if the experiences reported by the interviewees represent the norm for all polyamorous people. However, the results of this study add to the foundation of IMT and research on polyamorous communication for future studies by providing a base of comparison.

The method of the e-interviews reflected limitations in that it did not deliver as much depth of response as other qualitative methods, like one-on-one interview or focus group. The 
responses of the e-interviews were much more brief and less detailed than the phone interview responses. Another drawback of the method is the detachment of researcher and interviewee. For example, if an interviewee needed clarification of a question during an interview, they would ask me to repeat or rephrase the question. In the e-interviews, an interviewee would leave the question blank or write, "I don't understand." Additionally, as the e-interview questions were attached to a separate typed questionnaire, there may have been participant fatigue.

A potential limitation of the recruitment via the subReddits Polyamory and NonMonogamy is response bias from the repeated recruitment from the same pool of users. I recruited for the e-interviews and phone interviews with two separate postings at different times in the same subReddits. However, I did not ask the interview interviewees if they had participated in the e-interviews, so it is impossible to know if I received responses from the same people.

Additionally, my analysis was solely my own, and I did not illicit inter-coder reliability. Also within this limitation, I did not illicit member verification. Viability of the interpretation of the data is restricted to my own analysis, and could be strengthened with these authentications.

Another limitation of the sample was the racial background of interviewees. The present study contained 61 interviewees total between the e-interviews and the phone interviews, and 53 of the 61 interviewees were White/Caucasian. According to Sheff and Hammers (2010), this is a common limitation of sexuality studies on polyamorous communities. The authors provided justifications for the mostly white face of polyamorous communities, in that "the privilege of perversities" contains some social protections of those experiencing sexual stigmas. What this means is that those of privileged race and class would be more likely to participate in research regarding their relationship orientation. Plans for future research should contain methodological 
considerations for building samples and collecting data that will increase sample diversity and reflect the varied identities within the polyamorous community.

\section{$\underline{\text { Future Research }}$}

Future research regarding communication and polyamory is needed in that there are few studies within the field examining consensual non-monogamy or specifically polyamorous dynamics. There is a sizeable opportunity to open relational communication, health communication, and more intercultural communication to polyamory and the unique conversations between partners regarding negotiation, sexual health, discussion boundaries, and disclosures.

The present study served as a pivotal work in my research experience and inspired a variety of future analyses. The responses from interviewees, especially the interviewees, contained rich information regarding identity formation and negotiation of polyamorous individuals. Through the retrospective interview technique, many interviewees discussed their path to non-monogamy and the relational influences and models for non-monogamy discussions they sought out in developing their own identities. These paths included online forums (like Reddit), dating websites, blogs, social groups, romantic relationships, and recognizing internal feelings of non-monogamy. Additionally, future studies on relational negotiations and rules among polyamorous partners would be fitting for the field relational communication. As a common question received by interviewees was, “... and how does that work?” We could seek to explain in depth the communicative and relational rules in polyamorous relationships.

Although the topic did not arise in a significant way in the interviews, sexual health between polyamorous people is a particular interest of mine. I am interested in the 
communication between relationship partners regarding sexual health topics such as STI testing, protection, and contraception, as well as the communication polyamorous people and their doctors regarding their relational orientation and sexual health. 
APPENDIX A: IRB APPROVAL LETTERS 
University of Central Florida Institutional Review Board Office of Research \& Commercialization

12201 Research Parkway, Suite 501

Orlando, Florida 32826-3246

Telephone: 407-823-2901 or 407-882-2276

www research.ncf.edu/compliance/irb.html

\section{Approval of Exempt Human Research}

From: UCF Institutional Review Board $\# 1$

FWA00000381, IRB00001138

To: $\quad$ Allison T. Table and Co-PIs: Harry Weger \& Rachel Reymann

Date: July 06,2015

Dear Researcher.

On 07/06/2015, the IRB approved the following activity as human participant research that is exempt from regulation:

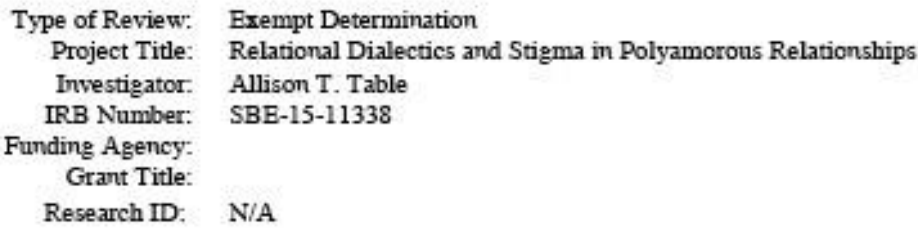

This determination applies only to the activities described in the IRB submission and does not apply should any changes be made. If changes are made and there are questions about whether these changes affect the exempt status of the human research, please contact the IRB. When won have completed vour research. please snbmit a Study Closure request in iRIS se that IRB records will be accurate

In the conduct of this research, you are responsible to follow the requirements of the Investigator Manual.

On behalf of Sophia Dziegielewski, Ph D., L.C.S.W., UCF IRB Chair, this letter is signed by:

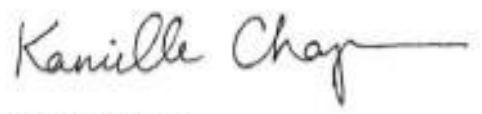

IRB Coordinator 


\author{
University of Central Florida Institutional Review Board \\ Office of Research \& Commercialization \\ 12201 Research Parkway, Suite 501 \\ Orlando, Florida 32826-3246 \\ Telephone: $407-823-2901$ or $407-882-2276$ \\ www.research.ucf.edu/compliance/irb.html
}

\title{
Approval of Exempt Human Research
}

From: UCF Institutional Review Board \#1

FWA00000351, IRB00001138

To: $\quad$ Allison T. Table and Co-PIs: Harry Weger \& Rachel Reymann

Date: $\quad$ March 09, 2016

Dear Researcher:

On 03/09/2016, the IRB approved the following activity as human participant research that is exempt from regulation:

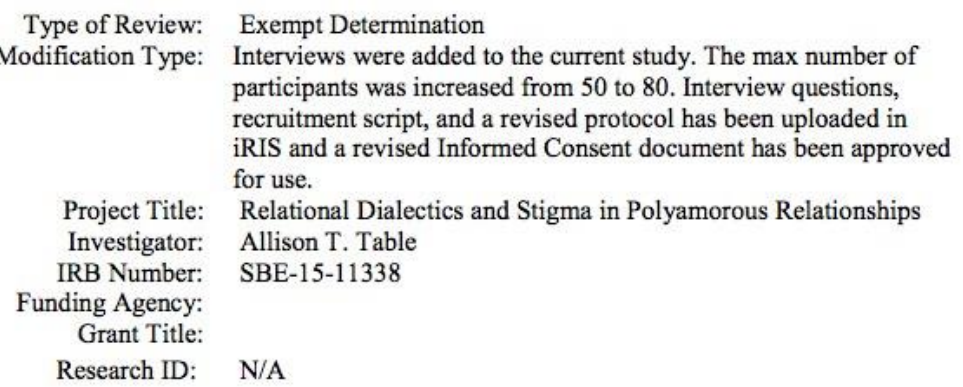

This determination applies only to the activities described in the IRB submission and does not apply should any changes be made. If changes are made and there are questions about whether these changes affect the exempt status of the human research, please contact the IRB. When you have completed your research. please submit a Study Closure request in iRIS so that IRB records will be accurate.

In the conduct of this research, you are responsible to follow the requirements of the Investigator Manual. On behalf of Sophia Dziegielewski, Ph.D., L.C.S.W., UCF IRB Chair, this letter is signed by:

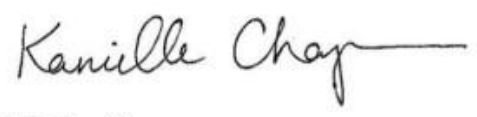

IRB Coordinator

Page 1 of 1 


\section{APPENDIX B: E-INTERVIEW QUESTIONS}


1. Age (Open Response)

2. What gender do you most identify with?

a. Woman

b. Man

c. Other (Open Response)

3. Which best defines your sexual orientation?

a. Gay

b. Straight

c. Lesbian

d. Bisexual

e. Pansexual

f. Queer

g. Asexual

h. Other (Please specify - Open Response)

4. What is your ethnicity?

a. White/Caucasian

b. Black/African American

c. Hispanic/Latino

d. Native American/American Indian

e. Asian/Pacific Islander

f. Indian

g. Other (Please specify - Open Response) 
5. Are you currently enrolled as a student?
a. Part-time graduate student
b. Full-time graduate student
c. Part-time undergraduate student
d. Full-time undergraduate student
e. No, I am not currently enrolled as a student

6. Which best describes your relationship orientation?
a. Polyamorous
b. Non-monogamous
c. Swinger
d. Monogamous
e. $\quad$ Other (Please specify)

7. In your own words, how do you define polyamory? (Open Response)

8. The following experiences inquire about being "out" and being polyamorous. The questions are about your good or bad experiences specifically. Who in your social circle (including friends, family, co-workers) knows about your polyamory? (Open Response)

9. What is the most insensitive thing someone has said to you about polyamory? (Open

Response)

10. How do you and your partners decide when to disclose your polyamory? (Open Response) 
11. Think about a time when you disclosed about your polyamory to someone new.

What factors do you consider in coming out as poly? (Open Response) 
APPENDIX C: INTERVIEW GUIDE 
○ This is Billy the researcher calling about our interview. How are you today?

○ Just give me a moment to set up my devices here. Hang on -

- (Once recording to have record of answer) Do I have permission to record?

- Keep in mind you can decline to answer any of these questions or stop the interview at any time. Your responses are confidential and you will not be identified by name in any reported data.

- Ok, before we jump in I just want to tell you a bit about my research purpose. My goal is to learn about polyamorous individuals' communication about their polyamorous identities, and also how that identity emerged. The study is for my master's thesis, and I aim to complete it by April. The completed manuscript will be sent to interviewees so you may see my findings and your contributions.

\section{Part 1, Introduction questions}

I have a couple demographic questions for you before we jump into the main interview questions.

- Your age, racial background, gender, pronouns, sexual orientation?

- How long have you been polyamorous?

- Describe your current relationships (relationship dynamics).

Part 2, Finding out about polyamory as a culturally situated identity

- How do you think polyamory is represented in society? 
- Can you recall a specific representation?

- What kinds of responses have you received?

- What are the most common questions you get, and how do you react to the questions?

- Can you give an example of how you would explain your relationships to someone new that you were disclosing it to (and felt comfortable disclosing it to)?

- What is the most insensitive thing someone has said to you about being poly?

- What are the possible negative consequences for these people finding out about being poly?

Part 3, Retrospective Interview Technique to uncover how polyamorous identity emerged

- Can you think of some turning points (either personal or relational) that brought you to identifying as polyamorous? What are they? (If needed: why are those significant?)

- How do you think (turning point 1,2 , etc.) contributed to how you view your relationships?

○ Can you recall a time when you first heard the word polyamory?

- Can you recall the first polyamorous person you met?

○ Can you recall the first time you called yourself polyamorous?

- What would you want people to know/understand most about your relationship identity? 
- What would you want people to know/understand most about polyamory in general?

- (And lastly,) Anything I haven't asked about that you think is critical to understanding poly identities or relationships? 


\section{APPENDIX D: REDDIT RECRUITMENT POST}




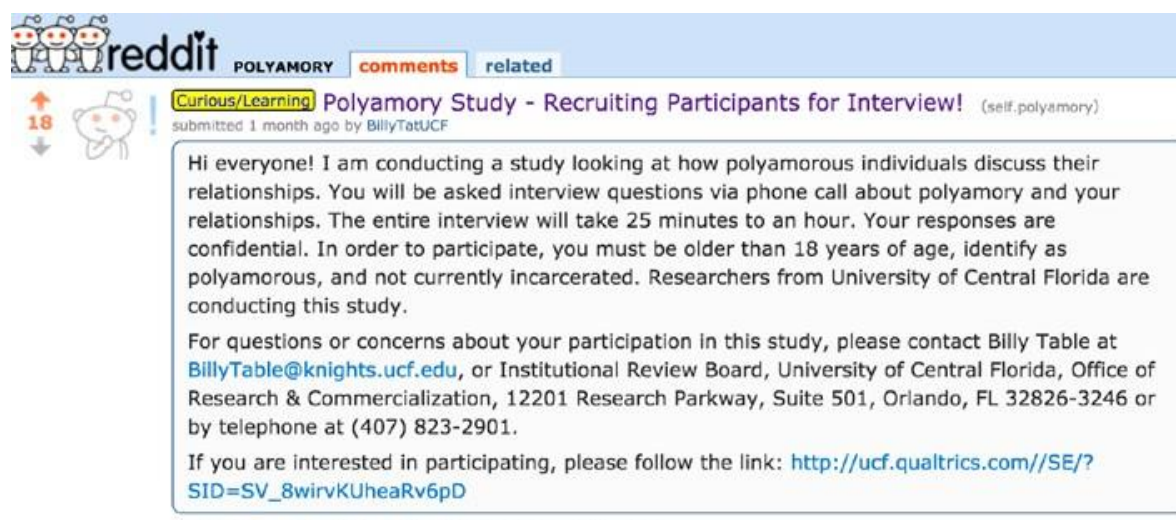

6 comments edit share save unhide delete nsfiw flair

all 6 comments
BillyTatuCF (1) |

search $Q$

this post was submitted on 16 Jan 2016

18 points ( $100 \%$ upvoted)

shortenic hetpos//rodd it $/ 419502$

Submit a new link

Submit a new text post

\section{polyamory}

unsubacribe 32,823 readers

117 users here now

Show my flair on this subreddit. It looks liket

Billyatucr (edit)

Welcome to /r/Polyamory!

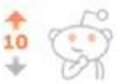

Recruiting participants for a polyamory study! (self.nonmonogamy lubmitted 1 month ago by BillyTatuCF

[Cross-posted to /r/polyamory]
Hi everyone! I am conducting a study looking at how polyamorous individuals discuss their
relationships. You will be asked interview questions via phone call about polyamory and your
relationships. The entire interview will take 25 minutes to an hour. Your responses are
confidential. In order to participate, you must be older than 18 years of age, identify as
polyamorous, and not currently incarcerated. Researchers from University of Central Florida are
conducting this study.
For questions or concerns about your participation in this study, please contact Billy Table at
BillyTable@knights.ucf.edu, or Institutional Review Board, University of Central Florida, Office of
Research \& Commercialization, 12201 Research Parkway, Suite 501 , Orlando, FL $32826-3246$ or
by telephone at (407) $823-2901$.
If you are interested in participating, please follow the link: http://ucf.qualtrics.com//SE/?
SID=SV_8wirvKUheaRv6pD

all 2 comments search

this post was submitted on 16 Jan 2016

10 points ( $100 \%$ upvoted)

shortink: https://redd.It/419vrj

Submit a new link

Submit a new text post

nonmonogamy

unsubscribe 11,802 readers

O 30 users here now

Show my flair on this subreddit. it looks like: BillyTatucF

Nonmonogamy is a blanket term covering several different types of interpersonal

relationships in which some or all participants have multiple marital, sexual, and/or romantic 
APPENDIX E: INFORMED CONSENT E-MAIL 


\section{Informed Consent}

Researcher: Billy Table, Nicholson School of Communication

Introduction: Researchers at the University of Central Florida (UCF) study many topics. To do this we need the help of people who agree to take part in a research study. You are being invited to take part in a research study which will initially include about 30 people in the United States or Canada. You have been asked to take part in this research study because you are polyamorous and are willing to discuss your relationships and identity. You must be 18 years of age or older to be included in the research study.

What you should know about a research study:

- Someone will explain this research study to you.

- A research study is something you volunteer for.

. Whether or not you take part is up to you.

- You can agree to take part now and later change your mind.

- Whatever you decide it will not be held against you.

- Feel free to ask all the questions you want before you decide.

Purpose of the research study: The purpose of this study is to collect polyamorous identity formation stories in order to better understand various relationship orientations beyond monogamy.

What you will be asked to do in the study: Billy will interview you about your polyamory. You will be asked about your personal experiences in being polyamorous and how you feel about it. 
Location/Time/Recording: The researcher will work with you to schedule a time for a phone interview. The interviews are expected to take approximately 25-60 minutes and they will be audio recorded. If you do not wish to be audio recorded, please inform the researcher. The digital file will be password protected on the researcher's private laptop computer. You may request a copy of the digital file.

Risks \& Benefits:

There are very minimal risks or discomforts involved in taking part in this study. The information you share may be considered personal and deeply emotional. You may experience some slight psychological discomfort if your polyamorous identity development was difficult. There is no specific expected benefit to you for taking part in this study. It does provide the opportunity to share your story and contribute to greater social understanding of a diversity of relationship orientations. There is no compensation or other payment to you for taking part in this study.

Study contact for questions about the study or to report a problem:

If you have questions, concerns, or additional comments please contact Billy Table at 407-257-4229 or BillyTable@ knights.ucf.edu. If you have any problems or complaints, you may contact the director of the Nicholson School of Communication at 407-8232683.

IRB contact about your rights in the study or to report a complaint: 
Research at the University of Central Florida involving human interviewees is carried out under the oversight of the Institutional Review Board (UCF IRB). This research has been reviewed and approved by the IRB. For information about the rights of people who take part in research, please contact:

Institutional Review Board, University of Central Florida, Office of Research \& Commercialization, 12201 Research Parkway, Suite 501, Orlando, FL 32826-3246 or by telephone at (407) 823-2901. You may also talk to them for any of the following: Your questions, concerns, or complaints are not being answered by the research team. You cannot reach the research team.

You want to talk to someone besides the research team.

You want to get information or provide input about this research. 


\section{REFERENCES}

Anderson, E. (2010). "At least with cheating there is an attempt at monogamy": Cheating and monogamism among undergraduate heterosexual men. Journal of Social and Personal Relationships, 27. 851-872. doi: 10.1177/0265407510373908

Barker, M., \& Langdridge, D. (2010). Whatever happened to non-monogamies? Critical reflections on recent research and theory. Sexualities, 13, 748-772. doi:

$10.1177 / 1363460710384645$

Barker, M. (2005). This is my partner, and this is my... partner's partner: Constructing a polyamorous identity in a monogamous world. Journal of Constructivist Psychology, 18, 75-88. doi: 10.1080/10720530590523107

Brown, P., \& Levinson, S. (1978). Universals in language usage: Politeness phenomena. Questions and politeness: Strategies in social interaction (56-311). Cambridge University Press.

Buzzfeed, [BuzzfeedYellow]. (2014, December 16). Ask a polyamorous person. Retrieved from https://www.youtube.com/watch?v=-o1gsI3e0u4

Collier, M. \& Thomas, M. (1988). Cultural identity: An interpretive perspective. Theories in intercultural communication. 99, 122-133. doi: 10.4135/9781412959384.n96

Conley, T., Moors, A., Matsick, J., \& Ziegler, A. (2013). The fewer the merrier?: Assessing stigma surrounding consensually non-monogamous romantic relationships. Analyses of Social Issues \& Public Policy, 13, 1-30. doi: 10.1111/j.1530-2415.2012.01286.x

Creswell, J., \& Creswell, J. (2007). Qualitative inquiry \& research design : choosing among five approaches. Thousand Oaks : Sage Publications, c2007. 
Crocker, J., Major, B., \& Steele, C. (1998). Social stigma. In D. T. Gilbert, S. T. Fiske, G. Lindzey, D. T. Gilbert, S. T. Fiske, G. Lindzey (Eds.), The handbook of social psychology, Vols. 1 and 2 (4th ed.) (pp. 504-553). New York, NY, US: McGraw-Hill.

Cupach, W. R., \& Imahori, T. T. (1993). Identity management theory: Communication competence in intercultural episodes and relationships. Intercultural Communication Competence, 112, 131-155. doi: 10.4135/9781483346267.n142

Emens, E. (2004). Monogamy's law: Compulsory monogamy and polyamorous existence. Review of Law and Social Change, 29. 277-376. doi: 10.2139/ssrn.506242

Duma, U. (2009). Jealousy and compersion in close relationships. Retrieved from: http://www.grin.com/en/e-book/137595/jealousy-andcompersion-inclose-relationships doi: 10.1037/e602052013-001

Farvid, P., \& Braun, V. (2013). Casual sex as 'not a natural act' and other regimes of truth about heterosexuality. Feminism \& Psychology, 23, 359-369. doi:10.1177/0959353513480018

Goffman, E. (1963). Stigma: Notes on the management of spoiled identity. Englewood Cliffs, N.J: Prentice-Hall. doi: 10.1136/pgmj.45.527.642

Goffman, E. (1967). Interaction ritual: Essays on face-to-face interaction.

Goldfeder, m. \& Sheff, E. (2013). Children of polyamorous families: A first empirical look. $J L$ and Social Deviance, 5. 150-243.

Gudykunst, W. B. (2003). Cross-cultural and intercultural communication. Thousand Oaks, CA: Sage Publications.

Gudykunst, W. B., Ting-Toomey, S., \& Chua, E. (1988). Culture and interpersonal communication. 
Haritaworn, J., Lin, C., \& Klesse, C. (2006). Poly/logue: A critical introduction to polyamory. Sexualities, 9, 515-529. doi: 10.1177/1363460706069963

Howard, S. C. (2012). Intercultural (mis) communication: Why would you "out" me in class?. Sexuality \& Culture, 16, 118-133.

Huston, T., Surra, C., Fitzgerald, N., \& Cate, R. (1981). From courtship to marriage: Mate selection as an interpersonal process. In S. Duck and R. Gilmour (Eds.) Personal relationships 2: Developing personal relationships (53-88). London and New York: Academic Press.

Im, E. \& Chee, W. (2012). Practical guidelines for qualitative research using online forums. Computers, informatics, nursing: CIN, 30. 604-610.

Imahori, T. T., \& Cupach, W. R. (2005). Identity management theory. Theorizing about intercultural communication, 195-210.

Klesse, C. (2006). Polyamory and its 'Others': Contesting the terms of non-monogamy. Sexualities, 9, 565-583. doi: 10.1177/1363460706069986

Klesse, C. \& Easton, D. (2006). The trials and tribulations of being a slut: Ethical, psychological, and political thoughts of polyamory. Sexualities, 9. 643-650. doi:

$10.1177 / 1363460706070006$

LaSala, M. (2004). Monogamy of the heart: Extradyadic sex and gay male couples. Journal of Gay \& Lesbian Social Services, 17, 1-24. doi: 10.1300/J041v17n03_01

Lee, P. W. (2006). Bridging cultures: Understanding the construction of relational identity in intercultural friendship. Journal of Intercultural Communication Research, 35, 3-22. doi: $10.1080 / 17475740600739156$ 
Lee, P. W. (2008). Stages and transitions of relational identity formation in intercultural friendship: Implications for identity management theory. Journal of International and Intercultural Communication, 1, 51-69. doi: 10.1080/17513050701690918

Link, B., \& Phelan, J. (2001). Conceptualizing stigma. Annual Review of Sociology, 27, 263-385. doi: 10.1146/annurev.soc.27.1.363

McLean, K. (2004). Negotiating (non)monogamy: Bisexuality and intimate partnerships. Journal of Bisexuality, 4. 83-97. doi: 10.1300/j159v04n01_07

Mint, P. (2004). The power dynamics of cheating: Effects on polyamory and bisexuality. Journal of Bisexuality, 4(3-4), 55-76. doi: 10.1300/J159v04n03_04

Mitchell, M., Bartholomew, K., \& Cobb, R. (2014). Need fulfillment in polyamorous relationships. Journal of Sex Research, 51, 329-339. doi:10.1080/00224499.2012.742998

Moors, A., Matsick, J., Ziegler, A., Rubin, J., \& Conley, T. (2013). Stigma toward individuals engaged in consensual non-monogamy: Robust and worthy of additional research. Analyses of Social Issues \& Public Policy, 13, 52-64. doi:10.1111/asap.12020

Moors, A., Conley, T., Edelstein, R., \& Chopik, W. (2015). Attached to monogamy? Avoidance predicts willingness to engage (but not actual engagement) in consensual nonmonogamy. Journal of Social and Personal Relationships, 32, 222-240. doi:

$10.1177 / 0265407514529065$

Orbe, M. (1998). Constructing co-cultural theory: an explication of culture, power, and communication. Thousand Oaks, CA: Sage.

Owen, W. F. (1984). Interpretive themes in relational communication. Quarterly Journal of Speech, 70, 274-287. doi:10.1080/10570318509374177 
Rambukkana, N. (2004). Uncomfortable bridges: The bisexual politics of outing polyamory. Journal of Bisexuality, 4, 141-154. doi: 10.1300/j159v04n03_11

Ritchie, A., \& Barker, M. (2006). 'There aren't words for what we do or how we feel so we have to make them up': Constructing polyamorous languages in a culture of compulsory monogamy. Sexualities, 9, 584-601. doi: 10.1177/1363460706069987

Robinson, M. (2013). Polyamory and monogamy as strategic identities. Journal of Bisexuality, 13, 21-38. doi:10.1080/15299716.2013.755731

Rhode, D. L. (2016). Adultery: Infidelity and the Law. Harvard University Press.

Sheff, E. (2005). Polyamorous women, sexual subjectivity, and power. Journal of Contemporary Ethnography, 34, 251-283. doi: 10.1037/e512142015-905

Sheff, E. (2011). Polyamorous Families, Same-Sex Marriage, and the Slippery Slope. Journal of Contemporary Ethnography, 40, 487-520. doi:10.1177/0891241611413578

Sheff, E. (2013). The polyamorists next door: Inside multiple-partner relationships and families. Rowman \& Littlefield. Journal of Contemporary Ethnography, 40, 487-520. doi: $10.1177 / 0891241611413578$

Sheff, E., \& Hammers, C. (2011). The privilege of perversities: race, class and education among polyamorists and kinksters. Psychology \& Sexuality, 2, 198-223. doi:10.1080/19419899.2010.537674

Smith, R. (2007). Language of the lost: An explication of stigma communication. Communication Theory, 17, 462-485. doi:10.1111/j.1468-2885.2007.00307.x

Ve Ard, C. \& Veaux, F. (2005). Polyamory 101. Retrieved from http://www.xeromag.com/poly101.pdf. 
Vogel, U. (1992). Whose Property? The double standard of adultery in nineteenth-century law. In Smart, C. (Ed), Regulating Womanhood: Historical essays on marriage, motherhood and sexuality. (pp. 147-165). New York: Routledge. doi:10.1017/s0047279400019681

Young, J. (2014). We are pioneers: Polyamorists' stigma management strategies. Master's thesis, Department of Sociology, Southern Illinois University Carbondale. Carbondale, IL. 